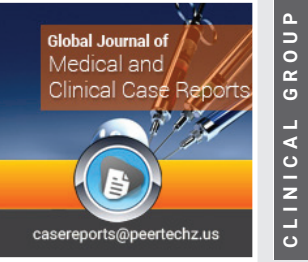

\section{Picking the right surgeon: Quantitative approach}

\section{E Suhir*}

Departments of Mechanical and Materials Engineering and Electrical and Computer Engineering, Portland State University, Portland, OR, ERS Co, 727 Alvina Ct, Los Altos, CA 94024, USA
Received: 10 December, 2021

Accepted: 21 December, 2021

Published: 22 December, 2021

*Corresponding author: E Suhir, Departments of Mechanical and Materials Engineering and Electrical and Computer Engineering, Portland State University, Portland, OR, ERS Co, 727 Alvina Ct, Los Altos, CA 94024, USA, Tel: 650.969.1530, 408-410-0886, E-mail: suhire@aol.com, e.suhir@ieee.org

Copyright License: (c) 2021 Suhir E. This is an openaccess article distributed under the terms of the Creative Commons Attribution License, which permits unrestricted use, distribution, and reproduction in any medium, provided the original author and source are credited.

https://www.peertechzpublications.com

\title{
Abstract
}

There is an abundance of useful verbal information and recommendations on how to make the best choice when referring a patient for surgery or when seeking the right surgeon for your own operation. A quantitative approach is suggested here on how this could be done through assessing the probability of success and/or the MeanTime-To-Failure (MTTF) of the planned operation by considering and comparing the skills of two highly qualified candidates. Then the chooser could continue this effort by comparing the background and the probability of success of the best one of these two candidates with the next suitable candidate, and then go on with the process for as many candidates as he/she would like to evaluate. The approach suggests using the double-exponential highly flexible and highly physically meaningful probability Distribution Function (DEPDF) as a suitable model. This function was introduced about a decade ago in the reliability physics to quantify, on the probabilistic basis, the outcome of a particular engineering, ergonomics or medical undertaking of importance. The surgeon's qualifications are identified in our approach as Human Capacity Factor (HCF). Figures of Merit (FoM) of this factor consider many relevant human qualities, as well as the durations and the outcomes of the surgeon's previous, both successful and failed, operations. The mental (cognitive) workload (MWL) reflects the complexity of the operation and, in the present analysis, is assumed to be the same for the two surgeon's considered. The role of an anesthesiologist is not taken into account directly in our approach: it is the surgeon who decides on his/hers partner, and the surgeon's choice is viewed as part of his/hers HCF. The general concepts are illustrated by a numerical example.

\section{Introduction}

There is, as is known, an abundance of useful verbal recommendations on how to choose the right surgeon when referring a patient for a surgery. Here are two more or less typical examples. S. Lewis [1] addressed knee replacement and suggested that the right orthopedic surgeon is found based on the following criteria: 1)he/she should be board certified in orthopedic surgery with specialization in knee replacements ("start by creating a list of potential orthopedic surgeons and continued with asking family, friends, and other healthcare providers for recommendations"); 2) has experience in treating patients with your specific condition, such as, e.g., knee arthritis ("research the doctors' credentials and experience, look for a knee doctor who performs knee replacements on a regular basis"); 3) practices at a hospital with high-quality outcomes in knee replacement or orthopedic surgery in general ("knee replacement surgery often requires a team of highly skilled and experienced healthcare providers"); 4) accepts your insurance ("to receive the most insurance benefits and pay the least outof-pocket for your surgery, you need to choose a knee surgeon that participates in your plan"); 5) you are comfortable talking with and who fully answers your questions ("...call each surgeon's office and ask for a consult appointment to meet and interview the doctor, ask yourself if... he or she respect your opinions and answer your questions in a way you understand"). P.M. Karpecki [2] suggested a "click away checklist" that can help to make the best choice when referring a patient for a cataract surgery: 1) confirm state credentials ("the Federation of State Medical Boards (FSMB) can tell you if the surgeon is licensed in your state"; 2) confirm surgical certification ("the American Board of Medical Specialties (ABMS) can tell you if the surgeon is board certified, i.e., has undergone lengthy training in a specialty and passed a stringent exam"; 3) uncover professional reprimands ("FSMB will provide the disciplinary history of specific doctors in any state"); 4) check 
ratings, number of procedures performed and complication rates ("ProPublica and Consumers Checkbook both have websites where they rate surgeons and provide information on the number of procedures and complication rates, based on recent Medicare data... To be fair to the surgeons, both adjust their results to allow for patients' health status, age and other factors, as some surgeons take on sicker or more frail patients").

In the analysis that follows we use these and other available descriptive, verbal, and, hence, non-quantified information as suitable input data for the suggested DEPDF model (see, e.g., [3-22]) to effectively organize and convincingly quantify, on the probabilistic basis, the most likely success of the planned operation performed by different available and experienced surgeons. Their professional and human qualities are identified in our approach as Human Capacity Factor (HCF). The Figures of Merit (FoM) of this factor consider many relevant human qualities, not only strictly professional, and particularly the durations of the surgeon's previous successful and failed operations. It is assumed, however, that the mental/cognitive Workload (MWL) reflecting the complexity of the planned operation, is the same for the surgeon's considered.

\section{Analysis}

Mental workload (MWL) vs. human capacity factor (HCF): Measuring the MWL has become, as is known, a key method of understanding and improving the role of the Human Factor (HF) in ergonomics engineering and human psychology, and there is an extensive published work on this subject (see, e.g., [9]). In this analysis it is assumed that the MWL is associated with the complexity of the anticipated operation and is the same for the two hypothetical surgeon's compared. HCF, unlike MWL, is a relatively new notion [3-6]. HCF plays with respect to the MWL approximately the same role as strength/ capacity plays with respect to stress/demand in structural analyses and in economics demand-vs-supply problems. HCF includes, but might not be limited to, the following major qualities that would enable a surgeon to successfully cope with an elevated MWL (see, e.g., [4,6]): age; fitness; state-of-health; psychological suitability for a surgical challenge; level of professional experience and qualifications; education, both special and general; relevant capabilities and skills; level, quality and timeliness of professional training; performance sustainability (consistency, predictability); independent thinking and acting; ability to concentrate, to anticipate, to withstand fatigue and to act in cold blood, when necessary; self-control and mature (realistic) thinking; ability to operate effectively under pressure, and particularly under time pressure, in a tireless fashion, for a long period of time (tolerance to stress); ability to make well substantiated decisions in a short period of time and quite often in uncertain environmental conditions; leadership ability; swiftness in reaction, when necessary; adequate trust (in assisting humans technologies, equipment, instrumentation); and, certainly, ability to maintain the optimal level of physiological arousal. To come up with suitable Figures-of-Merit (FoM) for the HCF, one could rank the above, and perhaps other qualities, on the scale from, say, one to ten, and calculate the average FoM for each surgeon and/or task. Clearly, MWL and HCF should use the same measurement units, which could be particularly non-dimensional (see Table 1 below). Special (accelerated) tests might be necessary to develop and conduct to establish the level of these qualities for surgeons, and particularly the average time for carrying out an operation. The importance of the relative levels of the MWL and the HCF in various otherthan-surgical Human-in-the-Loop (HITL) problems has been addressed and discussed in several earlier publications (see, e.g., $[6-10])$.

Double-Exponential Probability Distribution Function (DEPDF) for the application in question: We proceed from the following expression for the probability

$$
P=\exp \left[-\lambda t \exp \left(-\frac{F}{G}\right)\right]
$$

of non-failure of the planned surgical operation, when quantifying, on the probabilistic basis, its outcome and to select the most suitable surgeon to do it. Here $\frac{F}{G}$ is the ratio of

the surgeon's HCF, $F$, to the most likely MWL $G$ associated with the complexity of the operation and its possible consequences $t$, is the duration of the operation and $\lambda$ is thus far unknown sensitivity factor for this time. This factor could be determined assuming that the complexity $G=-\frac{F}{\ln \left(-\frac{\ln P}{\lambda t}\right)}$ of the

planned operation is independent of the particular surgeon's qualifications and skills (in effect, a surgical operation, as any other human activity, is somewhat less complicated for an individual of high HCF, but an assessment of this effect is of secondary importance and is beyond the scope of this analysis), i.e., of his/hers HCF, considering the professional records of two surgeons, \#1and \#2. Based on this assumption, we conclude that the following equation $-\frac{F_{1}}{\ln \left(-\frac{\ln P_{1}}{\lambda t_{1}}\right)}=-\frac{F_{2}}{\ln \left(-\frac{\ln P_{2}}{\lambda t_{2}}\right)}$ has to be fulfilled. This equation yields:

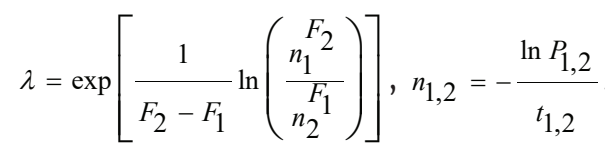

The distribution (1) makes physical sense. Indeed, the probability $p$ of non-failure increases with an increase in the ratio $\frac{F}{G}$ of the HCF to the most likely MWL and decreases with an increase in the "dimensionless time" $\lambda t$.

The entropy $H(P)=-P \ln P$ of the distribution (1) has its maximum $H_{\max }(P)=e^{-1}$ at the probability-of-non-failure $P=e^{-1}$. The corresponding time 
Table 1: Surgeon's Human Capacity Factor (HCF) Figures of Merit (example): Hypothetical ratings from one to ten.

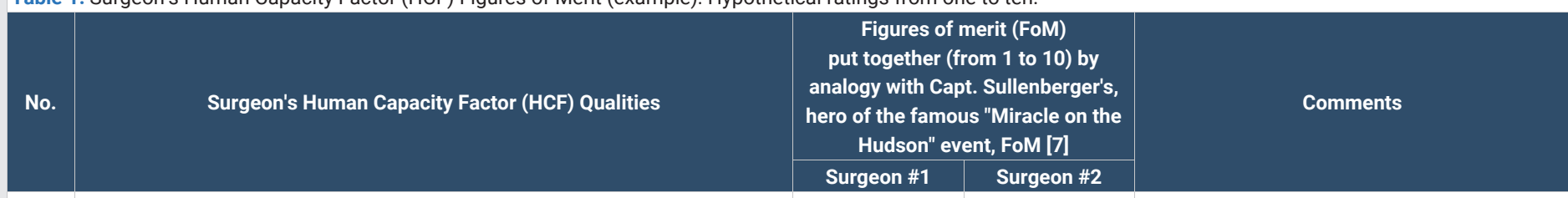

1 Possession of the American Board of Medical Specialties (ABMS) certification in the given field of surgery with particular specialization

2 Years of experience in treating patients in specific condition of importance

3 Practice at a hospital with known high-quality outcomes in the given field of surgery

Accepts your insurance and is comfortable to talk with State credentials: licenses in your state

Professional Federation of State Medical Boards (FSMB) reprimands (the disciplinary history of specific doctors), if any

Ratings, number of procedures performed and complication rates (could be found in "ProPublica and Consumers' Checkbook websites where they rate surgeons and provide information on the number of procedures and complication rates, based on recent Medicare data)

Performance sustainability/consistency/predictability: total number of operations and successful operations during the last ten years (if available)

Performance sustainability/consistency/predictability: total number of operations and failed operations during the last ten years (if available) Age (best age seems to be between, say, 45 and 65), fitness, general and current state of health

Psychological suitability for a surgical challenge and attributes, professional qualifications, experience, relevant capabilities and skills; were his/hers parents also surgeons or, if not, medical doctors at least?

Education, both special and general, quality and timeliness of professional 12 training (medical schools graduated from; continued professional training and courses, etc.)

13 Leadership ability, independent and mature (realistic) thinking and acting

14 Ability to anticipate ("expecting the unexpected") and to concentrate

15 High level of self-control and ability to act in cold blood ("cool demeanor") Tolerance to stress (ability to withstand fatigue, to operate effectively in a tireless fashion and for a long enough time; to act effectively under time 16 pressure and make well substantiated decisions in a short period of time and sometimes even in an uncertain conditions and to maintain the optimal level of physiological arousal)

17 Adequate trust (in assessing humans, technologies, equipment, instrumentation)

18 Average time to perform an operation Total Average HCF (F)

$$
\tau=\frac{1}{\lambda} \exp \left(\frac{F}{G}\right)
$$

can be considered, by analogy with the Arrhenius equation (see, e.g., [20]) in physical chemistry, as the Mean-Time to Failure (MTTF). Note, that the basic equation (1) could be written, considering (3), as

$$
P=\exp \left(-\frac{t}{\tau}\right)
$$

and that the time derivative of the probability (1) of nonfailure is

$$
\frac{d P}{d t}=-P \lambda \exp \left(-\frac{F}{G}\right)=\frac{P}{t}\left[-\lambda t \exp \left(-\frac{F}{G}\right)\right]=\frac{P \ln P}{t}=-\frac{H(P)}{t} .
$$

\begin{tabular}{c|c|}
\hline 10 & 10 \\
\hline 9 & 9 \\
\hline 9 & 9 \\
\hline 9 & 9 \\
\hline 9 & 9 \\
\hline & 10 (none) \\
\hline
\end{tabular}

9

8

9

10

10

10

10

10

8

9

8

9

156

8.6667

9

8

9

8

10
The first seven items are those indicated by S. Lewis [1] and P.M.Karpecki [2].

The figures of merit numbers are rather arbitrary. They are merely illustrations to the objective to show how critical human factors that are addressed, as a rule, as verbal and, hence, unquantifiable, could be nevertheless quantified on the probabilistic basis

"The entire life up to this moment was a preparation for this moment", Capt. Sullenberger

"I was sure I could do it", Capt. Sullenberger

"I am not just a pilot of that flight. I am also a pilot who has flown for 43 years..." Capt. Sullenberger

"We are going to be in the Hudson", Capt. Sullenberger

This information could presumably be obtained as a result of FOAT testing on a flight simulator (see [13])

$$
\text { See }[11,19]
$$

This relationship explains the physical meaning of the basic distribution (1): The time derivative of the probability of nonfailure is, in effect, the ratio of the entropy of this distribution to time.

\section{Numerical example}

Let surgeon \#1 (see Table 1) perform 100 operations and 92 of them were successful (by whatever criteria). The average duration of each operation, both successful or failed ones, was, say, $3 \mathrm{~h}=10,800$ s. Hence, $P_{1}=0.92$ and $t_{1}=10,800 \mathrm{~s}$. Then, using the notation in (2), we have: $n_{1}=\frac{-\ln P_{1}}{t_{1}}=\frac{-\ln 0.92}{10800}=7.7205 \times 10^{-6} s^{-1}$. Let surgeon \#2 perform 50 operations of the same type and complexity, and 45 of them were successful, so that $P_{2}=0.90$ Let the average duration of 
each operation, regardless of the outcome, was, say, $t_{2}=2.5 \mathrm{~h}$

$=9,000 s$. Then $n_{2}=\frac{-\ln P_{2}}{t_{2}}=\frac{-\ln 0.90}{9000}=11.7067 \times 10^{-6}{ }_{s}^{-1}$. Using

the first formula in (2) and the HCF FoMs from Table 1, we have:

$\lambda=\exp \left[\frac{1}{F_{2}-F_{1}} \ln \left(\frac{n_{1}}{F_{1}} n_{2}\right)\right]=\frac{1}{8.5000-8.6667} \ln \left(\frac{\left(7.7205 \times 10^{-6}\right)^{8.5}}{\left(11.7067 \times 10^{-6}\right)^{8.6667}}\right)=19,355.0 \mathrm{~s}^{-1}$

Then the MWL FoM can be found as

$$
G=\frac{F_{1}}{-\ln \left(\frac{n_{1}}{\lambda}\right)}=\frac{8.6667}{-\ln \left(\frac{11.7205 \times 10^{-6}}{19355}\right)}=0.40830
$$

, or, to make sure that there is no calculation error, as

$$
G=\frac{F_{2}}{-\ln \left(\frac{n_{2}}{\lambda}\right)}=\frac{8.5000}{-\ln \left(\frac{11.7067 \times 10^{-6}}{19355}\right)}=0.40830 \quad \text {. Then the two }
$$

MTTFs predicted by (3) are

$$
\tau_{1}=\frac{1}{\lambda} \exp \left(\frac{F_{1}}{G}\right)=\frac{1}{19355} \exp \left(\frac{8.6667}{0.40830}\right)=85442 \mathrm{~s}=23.734 h ;
$$

and

$$
\tau_{2}=\frac{1}{\lambda} \exp \left(\frac{F_{2}}{G}\right)=\frac{1}{19355} \exp \left(\frac{8.5000}{0.40830}\right)=56802 \mathrm{~s}=15.778 \mathrm{~h} ;
$$

No wonder that these MTTF are significantly longer than the times of operations (both surgeons are highly qualified) and that this time is appreciably larger for surgeon \#1, because his/hers HCF is higher.

\section{Discussion}

The author understands, of course, that the suggested approach is just an attempt to quantify things that are usually perceived as unquantifiable. The approach could be used in addition to, but never instead of, the natural, "oldfashioned", "verbal" analyses and considerations. Being, first of all, an applied mathematician, applied physicist and a materials scientist, the author believes that it is hard to improve anything, if the outcome of an undertaking of importance is not quantified, and that, because of various unpredictable intervening influences, such a quantification should be done on the probabilistic basis. The majority of the literature references in this paper reflect this consideration. This take is both a limitation and a strength of the approach. It is a limitation, because the reader is expected to have some knowledge of more or less elementary math (such as calculus and applied probability), and it is also a strength, because the reader/user, who possesses such knowledge, could benefit from using the suggested quantification-based judgment. In this connection I, the author, would like to quote the famous German physicist Heinrich Hertz, who said that "mathematical formulas have their own life, they are smarter than we, even smarter than their authors, and often provide more than what has been expected from them" and the great French mathematician Pierre-Simone Laplace, the founder of the applied probability theory, who indicated that this theory "is nothing but common sense reduced to calculation". So, the suggested approach strengthens, in the author's opinion, the common sense of the individual who tries to find the most suitable surgeon for the anticipated operation. As a matter of fact, two evaluation techniques are suggested in this paper. The first one is based on the average Human Capacity Factor (HCF) calculated in Table 1. This technique simply enables the chooser to consider different characteristics/"capacities" of the surgeons under consideration, but is unable to assess the probabilistic characteristics of the professional performance of the two individuals. This assessment is done by introducing a physically meaningful double-exponential probability distribution function (1), which provides an exhaustive, ultimate description of the variables of importance. The calculated "tau" value, the mean time to failure (MTTF), is a convenient characteristic of the ability of the surgeon to carry out a failure-free operation: if this time is significant, then there is a good reason to believe that the given surgeon handles the time effectively and skillfully, in a failure free fashion. As to the entropy, it is, as is known, an important characteristic of a probability distribution and, as follows from the formula (5), explains the physics underlying the basic distribution (1): The time derivative of the probability of human non-failure is proportional to this entropy and is inversely proportional to time. The HCF alone does not provide this physically important information, does it?

Will a patient be able to use the information addressed in this paper? Well, it depends on the patient. Someone like myself will. His/hers level of applied math could very well be higher than that of a surgeon. The math in this analysis is certainly within the framework of calculus and applied probability that are taught in universities. But even if the patient himself/ herself will not be able to use all the math that is considered in this paper, he/she would always be able to find someone who will be able to at least fill out the Table 1. As to the Mental Workload (MWL) G value, it is assumed in our analysis that this value reflects, first of all, the complexity of the operation, and this complexity, although depends on many factors, could be assumed to be independent of a particular surgeon and his/ hers HCF. It is true that the MWL level might be a little lower for a surgeon of high HCF, but this circumstance is viewed as one of a low importance, compared to the patient condition and, perhaps, other factors affecting the level of the complexity of the operation. The potential users of the suggested methodology could be advisors with a background, first of all, in a surgical profession. Perhaps, a special document should be developed based on the methodology, suggested in this paper.

\section{Conclusion}

A quantitative approach is suggested on how to evaluate probabilistic characteristics of a potential surgeon for a planned operation. It is shown that that could be done through 
assessing the probability of success and/or the MTTF of the planned operation for the two particular available highly qualified surgeons. Then the chooser could continue his/hers effort by comparing the background and the probability of success of the best of the two candidates with the next suitable one, and then continue the process for as many candidates as he/she would like to. The approach is based on the use of the double-exponential highly physically meaningful probability distribution function.

\section{References}

1. Marsa $L$ (2017) Act As If Your Choice Is Life or Death - Because It Often Is AARP Bulletin.

2. Karpecki PM (2020) How to Pick the Right Surgeon. Review of Optometry.

3. Suhir E (2010) Probabilistic Modeling of the Role of the Human Factor in the Helicopter-Landing-Ship (HLS) Situation. Int J Human Factor Modeling Simulation 1. Link: https://bit.ly/3qfGOR1

4. Suhir E (2012) Miracle-on-the-Hudson: Quantified Aftermath. Int J Human Factor Modeling Simulation 4.

5. Suhir E (2012) Human in the Loop: Predicted Likelihood of Vehicular Mission Success and Safety. J Aircraft 49. Link: https://bit.ly/3Ja8Q90

6. Suhir E (2014) Human-in-the-Loop: Probabilistic Predictive Modeling, Its Role, Attributes, Challenges and Applications. Theor Issues Ergon Sci 16: 99-123. Link: https://bit.ly/30Ld9q4

7. Suhir E (2015) Analytical Modeling Enables Explaining Paradoxical Situations in the Behavior and Performance of Electronic Materials and Products: Review. J Physical Mathematics 07.

8. Suhir E (2017) Human-in-the-Loop: Application of the Double Exponential Probability Distribution Function Enables Quantifying the Role of the Human Factor. Int J Human Factor Modeling Simulation 5: 354-377. Link: https://bit.ly/3eagHFt

9. Suhir E (2019) Human-in-the-Loop: Probabilistic Modeling of an Aerospace Mission Outcome. CRC Press.

10. Suhir E (2020) Quantifying Unquantifiable: the Outcome of a Clinical Case Must Be Quantified to Make it Successful. Glob J Medical Clin Case Rep 7: 123-129. Link: https://bit.ly/3ecMTYT
11. Suhir E (2019) Mental Workload (MWL) vs. Human Capacity Factor (HCF): A Way to Quantify Human Performance: Gregory and Inna Bedny eds., Applied and Systemic-Structural Activity Theory, CRC Press.

12. Suhir E (2019) Adequate Trust, Human-Capacity-Factor, ProbabilityDistribution-Function of Human Non-Failure and its Entropy. Int $\mathrm{J}$ Human Factor Modeling Simulation 7: 75-83. Link: https://bit.ly/3J6ssuW

13. Suhir E (2019) Assessment of the Required Human Capacity Factor (HCF) Using Flight Simulator as an Appropriate Accelerated Test Vehicle. Int Journal Human Factor Modeling Simulation (IJHFMS) 7: 71-74. Link: https://bit.ly/3poGeRX

14. Suhir E (2020) Quantifying Unquantifiable: the Outcome of a Clinical Case Must Be Quantified to Make it Successful. Glob J Medical Clin Case Rep 7 123-129. Link: https://bit.ly/3mmjgsu

15. Suhir E (2020) Risk-Analysis in Aerospace Human-Factor-Related Tasks: Review and Extension. J Aerosp Eng Mech 4: 265-272. Link: https://bit.ly/3J8cWhV

16. Suhir E (2020) Aerospace Electronics Reliability Must Be Quantified to Be Assured: Application of the Probabilistic Design for Reliability Concept. Int $\mathrm{J}$ Aeronautics Aerospace Res 7: 235-243. Link: https://bit.ly/3qedGtx

17. Suhir E (2020) Quanifying Unquantifyable in Aerospace Electronics and Ergonomics Engineering: Review. J Aerosp Eng Mech 4: 306-347. Link: https://bit.ly/3FglJuX

18. Suhir E (2020) Astronaut's Performance vs. His/Hers Human-Capacity-Facto and State-of-Health: Application of Double-Exponential-Probability-Distribution Function. Acta Astronaut 178

19. Suhir E, Paul G (2021) Probabilistic HSI Models. International Ergonomics Associate Triennial (IEA) Conf., Vancouver, CA, Track on Transport Ergonomics and Human Factor.

20. Suhir E (2021) Driver Propensity to Fatigue and Drowsiness: A Probabilistic Approach. Theor Issues Ergon Sci. Link: https://bit.ly/328tBBG

21. Suhir E (2021) Medical Undertakings Should Be Quantified to Have A Potentia to Be Improved. J Electron Sensors 4: 1-12. Link: https://bit.ly/327ZNoN

22. Laider KJ (1987) Chemical Kinetics, Third Edition, Harper \& Row 42. Link: https://bit.ly/3qj70PL

\section{Discover a bigger Impact and Visibility of your article publication with}

\section{Peertechz Publications}

\section{Highlights}

* Signatory publisher of ORCID

* Signatory Publisher of DORA (San Francisco Declaration on Research Assessment)

* Articles archived in worlds' renowned service providers such as Portico, CNKI, AGRIS, TDNet, Base (Bielefeld University Library), CrossRef, Scilit, J-Gate etc.

* Journals indexed in ICMJE, SHERPA/ROMEO, Google Scholar etc.

* OAI-PMH (Open Archives Initiative Protocol for Metadata Harvesting)

* Dedicated Editorial Board for every journal

* Accurate and rapid peer-review process

* Increased citations of published articles through promotions

* Reduced timeline for article publication

Submit your articles and experience a new surge in publication services (https://www.peertechz.com/submission).

Peertechz journals wishes everlasting success in your every endeavours.

Citation: Suhir E (2021) Picking the right surgeon: Quantitative approach. Glob J Medical Clin Case Rep 8(3): 120-124 